\title{
An Import Factor in Reticulocyte Lysates which Stimulates Processing of Several Precursors Destined for the Rat Liver Mitochondrial Inner Membrane*
}

\author{
Vigg Joste, ${ }^{a}$ Jean-Marc Berrez, ${ }^{b}$ Norbert Latruffe ${ }^{b}$ and B. Dean Nelson ${ }^{a}$

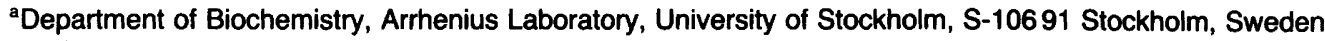 \\ and 'Laboratoire de Biochimie, Université de Franche-Comté, Besançon, Cedex, France
}

\begin{abstract}
Joste, V., Berrez, J.-M., Latruffe, N. and Nelson, B. D., 1987. An Import Factor in Reticulocyte Lysates which Stimulates Processing of Several Precursors Destined for the Rat Liver Mitochondrial Inner Membrane. - Acta Chem. Scand., Ser. B 41: 770-772.
\end{abstract}

Reticulocyte lysates contain a cytosolic factor which stimulates uptake and processing of precursor polypeptides by mitochondria in vitro..$^{1-3}$ The factor behaves like a protein in several respects. ${ }^{2-4}$ However, the identity of the cytosolic import factor and the mechanism by which it stimulates import/processing of precursor polypeptides remain unclear. The factor could not be demonstrated in dialyzed cytosol fractions from rat liver and kidney or from wheat germ extracts, ${ }^{2}$ but is present in an Escherichia coli extract. ${ }^{5}$ Furthermore, stimulation of import/processing by the reticulocyte cytosolic factor has so far been demonstrated with only one mammalian precursor polypeptide (the precursor to ornithine transcarbamoylase; EC 2.1.3.3).

In the present study we have shown that the reticulocyte factor is able to support processing of three additional rat precursors destined for the inner mitochondrial membrane. These results, together with results reported by Ohta and $\mathrm{Schatz}^{3}$ for yeast precursors, suggest a rather broad specificity of the factor. We also show that the lysate import factor does not stimulate processing by the isolated matrix fraction, indicating that a membrane event is involved.

\footnotetext{
* Communication at the Meeting of the Swedish Biochemical Society in Uppsala, August 21-22, 1987.
}

\section{Experimental}

In vitro translation. In vitro translation was carried out in nuclease-treated rabbit reticulocyte lysates ${ }^{6}$ programmed with rat liver free polysomes prepared as described by Ramsey and Steele. ${ }^{7}$ The composition of the translation medium was as described by Merrick. ${ }^{8}$ Translation was carried out for $60 \mathrm{~min}$ at $30^{\circ} \mathrm{C}$ in the presence of 30-40 $\mathrm{A}_{260^{-}}$-units of polysomal RNA per $\mathrm{ml}$ and ${ }^{35} \mathrm{~S}$-methionine (Amersham, $>1000 \mathrm{Ci} \mathrm{mmol}^{-1}$ ) at a final concentration of $1 \mathrm{mCi}$ per ml of translation mixture. Incorporation was terminated by removal of polysomes through centrifugation at $105000 \mathrm{~g}$ for $1 \mathrm{~h}$.

In vitro processing of precursor polypeptides with mitochondria and the mitochondrial matrix fraction. Labelled reticulocyte lysates were depleted of the cytosolic import factor by centrifugation through Sephadex G-25 equilibrated with $50 \mathrm{mM}$ Bicine $[N, N$-bis(2-hydroxyethyl)glycine; Sigma Chemical Company], $90 \mathrm{mM} \mathrm{KCl}, 5 \mathrm{mM}$ $\mathrm{KH}_{2} \mathrm{PO}_{4}$, $\mathrm{pH} 7.6 .^{9}$ Preparation of mitochondria and the mitochondrial matrix fraction, as well as precursor processing by mitochondria $(0.5 \mathrm{mg}$ protein $\mathrm{ml}^{-1}$ ) and by the isolated matrix fraction (1.6 $\mathrm{mg}$ protein $\mathrm{ml}^{-1}$ ) was performed as described by Kuzela et al. ${ }^{9}$ in a final volume of $500 \mu \mathrm{l}$. 
Samples were incubated for $60 \mathrm{~min}$ at $27^{\circ} \mathrm{C}$ and mitochondria were separated from lysates by centrifugation. The supernatant and pellet fractions were prepared for immunoabsorption according to Kuzela et al. ${ }^{9}$

Immunoabsorption, electrophoresis and fluorography. Immunoabsorption ${ }^{9}$ and sodium dodecylsulphate polyacrylamide slab gel electrophoresis $^{10}$ were performed as described. Fluorography was carried out with sodium salicylate-impregnated gels. ${ }^{11}$ Films were analyzed densitometrically with an LKB Ultroscan laser densitometer.

\section{Results}

The data in Fig. 1 show the effect of the reticulocyte lysate factor on the in vitro processing by mitochondria of the precursors to the $\beta$-subunit of $F_{1}$-ATPase (Fig. 1a, c), apo- $\beta$-hydroxybutyrate dehydrogenase (Fig. 1b,c) and the iron-sulfur protein of Complex III (Fig. 1c). Although the fluorographic image obtained with the iron-sulfur protein was too faint to reproduce photographically, it could be analyzed densitometrically, as were the images obtained for the $\beta$-subunit of $F_{1}$-ATPase and $\beta$-hydroxybutyrate dehydrogenase (Fig. 1c). In all three cases, the non-filtered lysate, containing the import factor, increased the amount of processed polypeptide sedimenting with mitochondria.

(a)

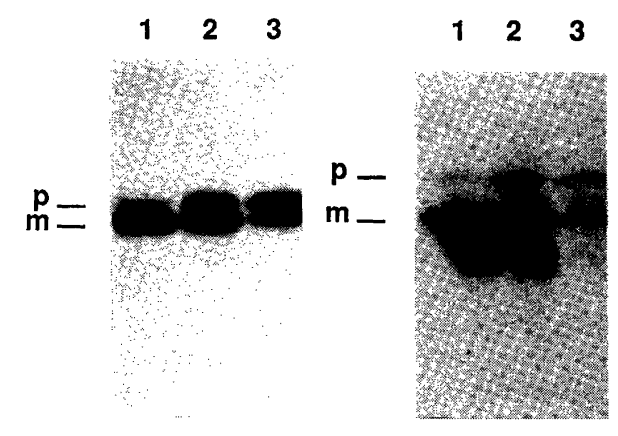

One way in which the lysate factor might act is by maintaining the precursor in a conformational state required for import or for cleavage by the matrix protease. To determine whether the lysate factor could stimulate proteolytic cleavage directly, processing was carried out with the isolated matrix fraction as described earlier ${ }^{9}$ (Fig. 2). Again, the fluorographic image obtained with the iron-sulfur protein was faint, but was analyzed densitometrically. The apparent low level of processing of pre-iron-sulfur protein by the matrix fraction (Fig. 2c) is probably due, at least in part, to the faint fluorographic image and the non-linear relationship between sample radioactivity and darkening of the film in this range. However, the results obtained for all three precursors indicate that the concentrations of nonfiltered lysate which stimulate uptake and processing by mitochondria do not influence processing by the isolated matrix fraction (Fig. 2).

\section{Discussion}

In the present study, import/processing by isolated mitochondria of the precursors to three topologically and functionally different mitochondrial inner membrane polypeptides has been shown to be stimulated by a reticulocyte lysate factor. Earlier similar observations, obtained with the precursor to the mitochondrial matrix polypeptide ornithine transcarbamoylase,,$^{1,2}$ have

(c)

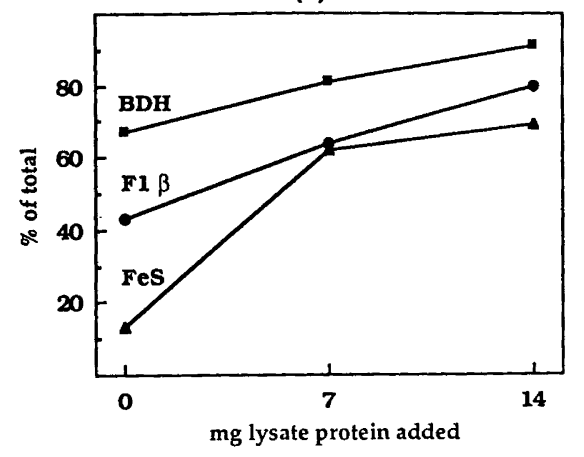

Fig. 1. Effect of a reticulocyte lysate factor on uptake/processing of precursors to mitochondrial inner membrane polypeptides by mitochondria. In vitro labelled and Sephadex G-25 filtered rabbit reticulocyte lysates were incubated with mitochondria and decreasing amounts of non-filtered, non-labelled lysate ( $28 \mathrm{mg}$

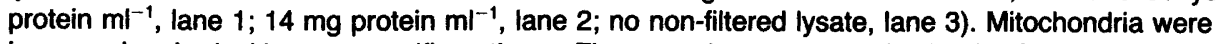
immunoabsorbed with monospecific antisera. Fluorographs are shown for (a) the $\beta$-subunit of $F_{1}$-ATPase and (b) $\beta$-hydroxybutyrate dehydrogenase; $m=$ mature, $p=$ precursor. (c) Quantitative measurement of the mature polypeptides formed was made by densitometric scans of fluorographs. $\mathrm{BDH}=\beta$-hydroxybutyrate dehydrogenase, $\mathrm{F} 1 \beta=$ the $\beta$-subunit of $\mathrm{F}_{1}$-ATPase, $\mathrm{FeS}=$ the iron-sulfur protein of Complex III. 
(a)

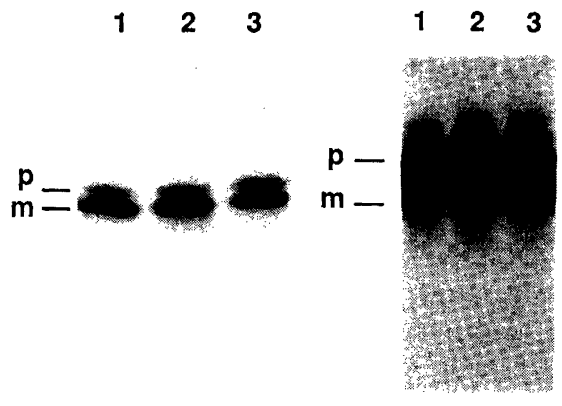

(b)

3 (c)

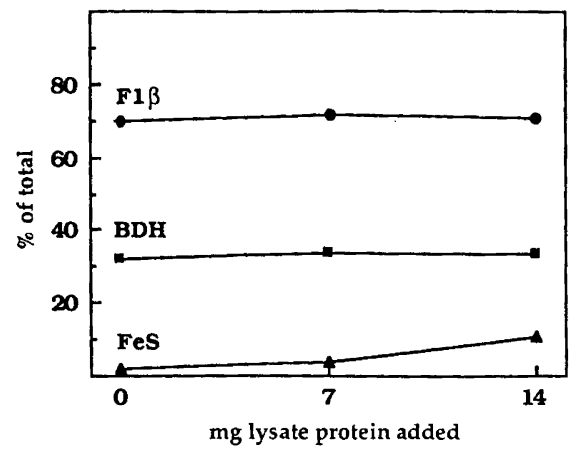

Fig. 2. Effect of a reticulocyte lysate factor on processing of precursors to mitochondrial inner membrane polypeptides by the mitochondrial matrix fraction. In vitro labelled and Sephadex G-25 filtered rabbit reticulocyte lysates were incubated with the mitochondrial matrix fraction and decreasing amounts of non-

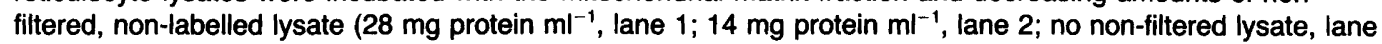
3). The incubation mixture was immunoabsorbed with monospecific antisera. Fluorographs are shown for (a) the $\beta$-subunit of $F_{1}$-ATPase and (b) $\beta$-hydroxybutyrate dehydrogenase; $m=$ mature, $p=$ precursor. (c) Quantitative measurement of the mature polypeptides formed was made by densitometric scans of fluorographs. $\mathrm{BDH}=\beta$-hydroxybutyrate dehydrogenase, $\mathrm{F}_{1} \beta=$ the $\beta$-subunit of $\mathrm{F}_{1}$-ATPase, $\mathrm{FeS}=$ the ironsulfur protein of Complex III.

thus been extended. A broad specificity of the lysate factor is therefore suggested.

Since import factors were not detected in the cytosolic fraction from rat liver and kidney or in wheat germ extracts, ${ }^{2}$ but were found in $E$. coli extracts, ${ }^{5}$ their role in the import/processing system is unclear. The trivial explanation that they merely stabilize the mitochondrial membrane has been discussed and rejected. ${ }^{3}$ Our findings suggest, however, that a membrane is required, since processing by the isolated matrix fraction is not influenced by reticulocyte lysate. The latter finding also seems to eliminate a direct role of the reticulocyte lysate factor in maintaining a protease-sensitive conformational state of the precursor. If the lysate factor is required for targeting precursors to mitochondria or for transport of precursors through the cytosol, as suggested by the studies of Argan and Shore ${ }^{4}$ the components involved must be well conserved since mammalian reticulocyte lysate also activates processing of yeast precursor polypeptides by yeast mitochondria. ${ }^{3}$ Furthermore, $E$. coli extracts also contain a factor which supports processing of a mammalian precursor polypeptide by mammalian mitochondria. ${ }^{5}$

The picture which emerges from the present study is that the reticulocyte lysate factor has a broad specificity and is capable of promoting processing of the precursors to a variety of un- related inner membrane proteins through a mechanism which requires a membrane.

Acknowledgements. This study was supported by funds from the Swedish Natural Science Research Council (B.D.N.) and the Swedish Institute (J.-M.B.). The technical assistance of B. Persson is gratefully acknowledged. The authors thank M. S. El Kebbaj, P. Adami and R. Duroc for assistance in production of the anti- $\beta$-hydroxybutyrate dehydrogenase antisera.

\section{References}

1. Argan, C., Lusty, C. J. and Shore, G. C. J. Biol. Chem. 258 (1983) 6667.

2. Miura, S., Mori, M. and Tatibana, M. J. Biol. Chem. 258 (1983) 6671.

3. Ohta, S. and Schatz, G. EMBO J. 3 (1984) 651.

4. Argan, C. and Shore, G. C. Biochem. Biophys. Res. Commun. 131 (1985) 289.

5. Sheffield, W. P., Nguyen, M. and Shore, G. C. Biochem. Biophys. Res. Commun. 134 (1986) 21.

6. Pelham, H. R. B. and Jackson, R. J. Eur. J. Biochem. 67 (1976) 247.

7. Ramsey, J.C. and Steele, W. J. Biochemistry 15 (1976) 1704.

8. Merrick, W. C. Methods Enzymol. 101 (1983) 606.

9. Kuzela, S., Joste, V. and Nelson, B. D. Eur. J. Biochem. 154 (1986) 553.

10. Laemmli, U. K. Nature (London) 227 (1970) 680.

11. Chamberlain, J. P. Anal. Biochem. 98 (1979) 132. 